\title{
LES BOISSONS DE « DÉFENSE » EN HONGRIE
}

\section{FRUITS ET SOUS-PRODUITS DU LAIT}

\author{
par \\ L. TELEGDY KOVATS, E. SZILAS, H. BRUCKNER
}

Université Polytechnique de Budapest.

\begin{abstract}
Les boissons hygiéniques de "defense" ont pour rôle d'augmenter la résistance de ceux qui travaillent dans des conditions nuisibles à la santé et de diminuer les effets nocifs de celles-ci. Se basant sur des considérations théoriques et des observations pratiques, la loi garantit en Hongrie aux travailleurs de force (par ex. dans les industries chimiques, les entreprises "chaud-froid", souterraines, etc...) la distribution comme aliments et boissons de "défense" d'abord du lait, des marmelades de fruits, de raisin, ensuite de thé russe, thé de cynorrhodons, eau gazeuse et biscuits salés. Leur effet s'avère bon (par ex. au point de vue de la teneur en vitamines, en substances minérales, comme rafraîchissants, etc...) mais leur composition n'est pas assez variée et leur mise en circulation difficile. Il serait donc souhaitable d'élaborer une technologie de boissons de défense présentant des saveurs plus variées, une valeur nutritive plus grande, et un prix de revient moins élevé, ce qui pourrait permettre une distribution plus rationnelle.
\end{abstract}

On peut fabriquer des boissons de défense nouvelles, d'un goût agréable, en utilisant ceux des sousproduits de l'industrie laitière tels le petit lait et le babeurre, qui jusqu'à présent n'ont pas servi d'aliment sur une grande échelle, leur technologie à l'échelon industriel n'étant pas au point. L'utilisation de telles boissons de défense peut être d'une grande importance économique et hygiénique. C'est pour cette raison que nous nous sommes donné comme tâche d'élargir le choix des boissons de défense utilisées actuellement et de réaliser de nouvelles boissons avec les sous-produits de l'industrie laitière en tenant compte de la teneur en vitamines des éléments nutritifs, des calories, de la digestibilité. Nous nous sommes assigné comme autre but de trouver un emballage bon marché et hygiénique pour les nouvelles boissons et d'assurer l'entreposage dans les meilleures conditions.

L. T. K. 


\section{Lu dans Fruits, il y a 50 ans ...}

\section{Substances de base et substances auxiliaires utilisées.}

Comme substances de base nous avons utilisé le lait, le petit lait et le babeurre.

Le lait contient des éléments nutritifs nécessaires à 1 a vie dans une proportion favorable et sous une forme facilement digestible (I) les acides aminés essentiels y sont représentés, les graisses du lait sont directement absorbées par le tube digestif. En résumé le lait peut être considéré comme un aliment très complet, mais il est pauvre en fer.

Le petit lait est un sous-produit de 1'industrie du fromage. Sa composition est déterminée par son origine. Le petit lait obtenu avec la présure contient davantage de lactose et de protides; le petit lait obtenu par acidification contient moins de lactose, davantage d'acide lactique et de substances minérales. L'utilisation du petit lait pour la préparation de boissons de défense se justifie surtout par sa forte teneur en hydrates de carbone et en sel (2).

Le babeurre est un liquide qui subsiste après le barratage de la crème. Sa composition correspond en général à celle du lait écrémé. Il est encure plus facile à digérer que le lait et les autres produits laitiers, en raison de la présence d'une telle caséine qui se dénature dans l'estomac en de petits flocons fins facilement décomposables. La valeur nutritive du babeurre est encore augmentée par la forte teneur en lécithine $(0, \mathrm{I}-0,8 \%)$. Cette forte teneur s'explique par le fait que pendant le barratage la lécithine de la crème passe presque entièrement dans le babeurre. L'inconvénient du babeurre réside dans son peu d'homogénéité; en effet le sérum se sépare facilement du coagulum.

Les consommateurs hongrois n'attachent généralement pas au babeurre l'intérêt qu'il mériterait en tant qu'aliment populaire et boisson rafraîchissante. Il existe par contre des pays où la demande de babeurre est telle que le produit naturel ne suffit pas à la consommation et qu'on produit du babeurre artificiel. La consommation du babeurre peut et doit être augmentée par une propagande adéquate dans tous les domaines et notamment en augmentant le choix des boissons de défense.

Les substances auxiliaires utilisées ont été les suivantes :

La tomate. Ses composants les plus précieux sont : des sucres, des acides organiques (acide citrique, malique, tartrique, lactique), la vitamine C. Les teneurs en provitamine $\mathrm{A}$ et en vitamine $\mathrm{B}$ sont également importantes. L'effet rafraîchissant des acides et les diverses vitamines rendent la tomate apte à compléter les boissons de défense.

Les épinards, l'oseille. Ces deux légumes se distinguent des autres variétés de légumes par une forte teneur en fer.
Ainsi la faible teneur en fer du lait peut être compensée par une combinaison des légumes ci-dessus. On trouve également en quantités importantes dans les épinards les sels de calcium et dans l'oseille les acides organiques. Le fait que ces deux légumes puissent être disponibles dès le début du printemps constitue un atout supplémentaire pour leur utilisation. Les matières colorantes naturelles contenues dans les épinards et l'oseille (chlorophylle) contribuent également à rendre les boissons de défense attrayantes par leur aspect extérieur.

La carotte, le persil, le céleri. Tous les trois ont une forte teneur en vitamines. Notamment la teneur en carotène de la carotte est élevée.

La betterave rouge. Sa valeur dans la nutrition est représentée par le sucre $(6-9 \%)$ et la vitamine C. Elle peut être utilisée avantageusement moins en raison de son goût que comme substance colorante.

La pomme. Sa teneur en vitamines et en substances minérales, son goût agréablement acidulé et son effet rafraîchissant en font un fruit tout à fait indiqué pour une boisson de défense.

Le citron. Les vitamines $\mathrm{C}$ et $\mathrm{P}$ contenues dans le citron jouent un rôle dans l'augmentation de la résistance de l'organisme. Ses huiles essentielles et ses acides organiques lui confèrent un arôme agréable. Nous avons cherché à remplacer le citron par un fruit hongrois (le cynorrhodon) qui présente des qualités analogues que nous exposerons ci-après.

La griotte, la groseille, la tramboise, la fraise. L'utilisation de ces fruits est saisonnière. Elle s'impose en raison de leur teneur en vitamines, sucre, acides et arôme, de leur effet rafraîchissant et de leur couleur.

Le cynorrhodon. Un fruit sauvage ayant un goût acide et une teneur en vitamines seize fois plus grande que le citron. Ce fruit convient bien pour aromatiser les boissons de défense. Il a l'inconvénient de ne pas être cultivé systématiquement.

Le thé. Il est à côté du café le plus important des aliments contenant des bases puriques. Ses substances acides sont la caféine et la théine $(0,5-2 \%)$. Les feuilles contiennent également des huiles essentielles aromatiques. Convient pour des boissons de défense dans lesquelles la caféine .est parfois nécessaire.

La chataigne, l'amande. Ces fruits ont un goût si agréable 


\section{Lu dans Fruits, il y a 50 ans ...}

qu'il est possible de faire adopter à des jeunes n'aimant pas le lait des boissons de défense aromatisées aux châtaignes ou à l'amande.

Les matières ci-après ont été également utilisées, mais en si faibles quantités qu'il est inutile d'en énumérer les qualités. Ce sont le sucre, le sel, la viande de bœuf (bouillon), la semoule, la poudre d'œuf, les oignons, l'ail, le raifort, la moutarde, le poivre, la muscade, le cumen, la vanille et l'anis.

\section{Emballage en feuilles de matières plastique.}

Mousse de polyéthylène (feuille d'essai de la maison munichoise Alkor). Ce matériau consiste en polyéthylène plissé inséré entre deux membranes minces de polyéthylène. D'apparence agréable, donnant l'impression de la cire au toucher, il se présente comme une substance blanche, opaque et de $0,35 \mathrm{~mm}$ d'épaisseur.

$N . H . N$. polyéthylène. Type de polyéthylène fabriqué à basse et haute pression, à triple couche ; feuille transparente de $0,070 \mathrm{~mm}$ d'épaisseur (autre feuille d'essai de la maison Alkor).

$H$. N. polyèthylène. A double couche, feuille transparente de $0,030 \mathrm{~mm}$ d'épaisseur.

Hostalène. Il s'agit d'une fabrication de la maison
" Kalli » de Wiesbaden. D'apparence opalescente, opaque, facilement stérilisable. Nous l'avons utilisé pour nos essais d'entreposage en 0,085 et $0,040 \mathrm{~mm}$ d'épaisseur.

Cellothène. Feuille à double couche de $0,070 \mathrm{~mm}$ d'épaisseur formée d'une combinaison de polyéthylène et de cellophane. Convient parfaitement à un bon emballage pratique et économique pour des denrées alimentaires, des liquides, des graisses, des substances aromatiques, etc.

Feuille de cellophane. N'a été utilisée qu'en référence pour la comparaison des mesures.

Ces feuilles d'essais plastiques présentent toutes l'avantage de pouvoir être dessoudé le long de leur ligne de soudure au contact de la chaleur.

\section{Technique de la préparation des boissons de défense.}

Dans nos travaux techniques (composition des mélanges) nous nous sommes basés principalement sur nos propres conceptions, la littérature existante ne présentant que quelques indications schématiques. Ont servi :

Pour l'aromatisation des boissons de défense à base de lait : les fruits, les carottes, les amandes, les châtaignes, la poudre d'œuf, la semoule, le thé russe, le cynorrhodon.

Pour l'aromatisation des boissons de défense à base de petit lait les fruits et le miel.

Pour l'aromatisation des boissons de défense à base de babeurre le bouillon de viande, les fines herbes, les condiments.

Boissons à base de lait.

Lait aux fruits. Pour la préparation de cette boisson nous avons utilisé les fruits sous forme liquide. Après avoir coupé les fruits en parcelles, nous avons passé cellesci à la centrifugeuse et avons obtenu en quelques secondes du jus. De cette manière nous avons pu utiliser par ex. $60 \%$ des fraises, $55 \%$ des pommes. Après le mélange du lait et du jus de fruits (fraises, framboises, pommes, cynorrhodons) le jus insuffisamment sucré fut additionné de sucre cristallisé (saccharose). Pour le sirop de framboises ou de cynorrhodons qu'on trouve déjà sucré dans le commerce, une addition de sucre n'était plus nécessaire. Pour augmenter l'effet rafraîchissant on saturait avec du gaz carbonique. Aux fraises on ajoutait de l'eau gazeuse.

Boissons aux amandes et aux châtaignes. La fabrication de ces boissons demande une technique particulière. Pour la préparation du lait aux châtaignes, celles-ci étaient d'abord mises à l'étuvée, puis nettoyées et réduites en purée, et additionnées de vanille et de sucre. Après avoir fait bouillir un quart du lait nous avons fait une bouillie du tout, puis ajouté le lait froid pour réduire au minimum l'altération des substances actives si précieuses.

Pour la confection du lait aux amandes nous avons commencé par enlever la peau brune des amandes après les avoir passées à l'eau chaude. Puis nous les avoris grillées pour leur faire obtenir une couleur brun clair et nous les avons réduites en poudre fine. Pour la suite de l'opération nous avons procédé comme pour le lait aux châtaignes. 


\section{Lu dans Fruits, il y a 50 ans ...}

Lait aux carottes. Le jus de carottes est obtenu de la même façon que le jus de fruits avec possibilité d'utilisation de la carotte à $45 \%$. La préparation de la boisson de défense est la même que celle du lait aux fruits. La seule différence consiste dans 1'addition d'acide citrique après le sucrage. De cette façon on obtient presque le même effet de goût que pour les jus de fruits.

Lait aux oufs. On aromatise des jaunes d'œufs crus et sucrés avec de la vanille puis on y verse du lait chaud. Dans l'intérêt de la fabrication industrielle nous avons élaboré un deuxième procédé pour éviter la perte des blancs d'œufs : avec de la poudre d'œufs, du sucre, de la vanille et un quart de lait chauffé on fait une bouillie claire à laquelle on ajoute le reste du lait froid.

Lait aux flocons de semoule. D'un peu de lait et de semoule on fait une bouillie épaisse en veillant à ce que, après la cuisson, les grains gonflés forment une bouillie homogène. On dilue ensuite le produit avec du lait sucré tout en remuant jusqu'à ce qu'on obtienne une consistance buvable.

Lait au thé. Nous avons aromatisé le lait avec du thé provenant d'essence de thé concentré dix fois. L'essence préparée avec de l'eau chaude comme le thé est ensuite sucrée et diluée avec du lait.

Boissons à base de petit lait.

La plus grande difficulté dans la préparation de boissons de défense à base de petit lait consiste à assurer la précipitation de la matière protéique et à éliminer l'odeur caractéristique du petit lait. D'après les indications de la littérature (4) la matière protéique précipite par addition d'acide tanique. Elle peut être enlevée par un filtrage répété. L'emploi du charbon actif constitue une autre méthode (5). Reiter a mis au point le procédé dit « Moltra " (6) par lequel l'odeur désagréable du petit lait disparaît en cultivant dans le petit lait une moisissure du groupe Geotrichum. Cette moisissure fait fermenter 1'acide lactique mais non le lactose. $\mathrm{Au} \mathrm{pH}$ de 3,9 le petit lait ne contient pas de matière protéique précipitée. D'après des indications soviétiques, la technique la plus simple pour l'élimination des protides est la précipitation par chauffage et filtration. Appliquant le procédé soviétique dans nos recherches nous avons donc précipité les matières protéiques par chauffage et les avons séparées au moyen de charbon actif qui avait été humecté au préalable avec du petit lait. On peut obtenir ainsi un sérum de petit lait transparent, ayant une odeur et un goût agréable.

Petit lait aux fruits. Pour la fabrication du petit lait aux fruits nous avons préparé les griottes et les groseilles de la manière indiquée ci-dessus. La préparation correspond à celle décrite pour le lait aux fruits.

Pour la préparation du petit lait au citron nous avons ajouté non seulement du jus de citron, mais aussi des fragments d'écorce, ce qui assure un très bon arôme. La boisson préparée de cette façon a bien un goût agréable, mais sa couleur n'est pas attrayante. Aussi a-t-on utilisé comme colorant artificiel le jaune " alimentaire " (le 4-aminobenzol 3,4 disulfonate de sodium à raison de $7 x, 5 \%$ de produit pur).

Pour les besoins industriels on mit au point une boisson dans laquelle le citron était remplacé par de l'acide citrique et de l'acide ascorbique.

Les boissons de petit lait et de cynorrhodons étaient préparées avec du sirop de cynorrhodons ou du jus de baies séchées de la même façon que le lait aux cynorrhodons.

Petit lait au miel. En aromatisant du petit lait avec du miel et de l'anis nous avons obtenu une boisson de défense d'un goût et d'une valeur nutritive tout particuliers. Le miel était dissous dans une partie du petit lait, de l'anis moulu était ajouté à la boisson et le tout filtré trois heures après.

\section{Boissons de défense à base de babeurre.}

Dans la préparation de boissons à base de babeurre 1'hétérogénéité du babeurre constitue un inconvénient majeur. Aussi est-il indiqué d'agiter le babeurre avant usage. Pour éviter la disjonction du mélange on y ajoute les colloïdes de protection par ex. $0,25 \%$ de gélatine. Pour certaines boissons le babeurre a été utilisé dans sa forme clarifiée. D'après les expériences soviétiques la clarification a été faite de la façon suivante : les albumines ont été séparées par floculation puis filtrées. Le babeurre clarifié a été également employé pour des boissons qui ont été aromatisées avec des épinards, de l'oseille, de la betterave rouge et de la purée de paprika (vitapric), afin de mettre plus en évidence la couleur rouge ou verte des boissons.

Babeurre au jus d'épinards et d'oseille. Seuls les jus obtenus par une centrifugeuse " Progress " furent utilisés. Pour augmenter le goût agréable, ces deux boissons étaient non seulement saturées de gaz carbonique mais aussi additionnées d'acide citrique.

Babeurre an jus de betterave rouge. On utilisa le jus de betteraves rouges cuites. Le goût douceâtre peut être compensé par du jus de citron et du cumin pilé. On dilue ensuite avec de l'eau et on sature à l'aide de gaz carbonique.

Babeurre au jus de tomate. Il est préparé avec de la purée de tomates, légèrement sucré et additionné d'eau gazéifiée. C'est une boisson des plus agréables.

Avec des boissons composées de carottes, persil et célevis nous avons obtenu des effets de goût très prononcés en 


\section{Lu dans Fruits, il y a 50 ans ...}

utilisant des substances complémentaires telles le jus de citron, le jus de pommes, la moutarde, le vitapric, la purée de tomates, le jus de raifort, le jus d'oignons. Malgré ces effets de goût intéressants nous ne conseillons pas de les introduire.

Soupes chaudes au babeurre.

a) Du bouillon de viande préparé avec des légumes, de la viande et des os et mélangé à chaud avec du babeurre. Comme condiments nous nous sommes servis de muscade et de poivre en petites quantités. Cette boisson se distinguait parmi les boissons de défense à base de babeurre par sa valeur nutritive et son goût. b) Une soupe semblable mais encore plus riche peut être préparée en utilisant les légumes bouillis et réduits en purée.

Les deux soupes au babeurre doivent être prises chaudes. En dehors du goût et de la valeur nutritive la soupe au babeurre présente l'avantage d'utiliser les os qui comme le babeurre et le petit lait sont considérés comme des déchets difficilement utilisables.

Babeurre aux vitamines. En ajoutant du vitapric et du jus de raifort au babeurre on obtient une teneur élevée en vitamines, une couleur agréable et un bon goût qui peut encore être augmenté par l'addition d'acide citrique et d'eau gazéifiée.

\section{Contrôle.}

L'étude du contrôle fut effectuée sur les trois points suivants :

r. Fixation de la composition et de la qualité des boissons de défense.

2. Constatation de la qualification des feuilles plastiques comme matière d'emballage.

3. Recherche des meilleures conditions d'entreposage.

\section{Composition et qualité.}

I) pour établir le poids spécifique nous avons utilisé des aéromètres. La mesure fut faite à $15^{\circ} \mathrm{C}$;

2) le dosage de la matière grasse a été fait par la méthode de Gerber;

3) le dosage de la teneur en sucre fut réalisé Đar la méthode polarimétrique ;

4) le dosage des extraits secs a été réalisé paf l'analyse du poids. Les résultats furent donnés en pourcentage de poids ;

5) le dosage des matières protéiques fut fait par la méthode de Kjeldahl;

6) le degré d'acidité a été établi par titration à haute fréquence d'après Pungor. Cette méthode semble particulièrement convenir au dosage de l'acidité des liquides colloïdaux colorés $(7,8)$;

7) dosage de la vitamine $C$. Nous avons étudié plusieurs méthodes :

a) la méthode de Tilman : le virage n'était pas assez net dans le cas des liquides colorés (9);

b) la méthode de Schulek, modifiée par $\mathbf{M}^{\mathrm{mes}}$ Kiszel et Lanyi (ro). L'essentiel de cette méthode consiste dans l'addition d'une combinaison ferrique et ensuite de $\alpha$, $\alpha$ '-Dipyridyl à l'extrait de la matière à examiner. L'acide ascorbique de l'extrait réduit les ions ferriques en ions ferreux qui donnent avec le dipyridyl une liaison complexe soluble avec apparition d'une couleur rouge. L'intensité de la couleur obtenue est proportionnelle à la quantité d'acide ascorbique ;

c) méthode de Effer : le dosage se fait au moyen du bleu de méthylène. La teneur en vitamine $\mathrm{C}$ se trouvant dans le lait diminue pendant que le lait repose en raison de l'oxygène dissous et est donc difficile à doser d'une façon précise. Aussi B. Wilberg produit-il, avec de l'acide oxalique, à partir du lait, un sérum tel qu'il diminue ou annihile dans certains cas l'effet de l'oxygène dissous (II).

Des trois méthodes essayées nous avons retenu celle de Effer comme la plus appropriée, le virage se fait en effet rapidement et nettement (de l'incolore au bleu) et l'effet perturbateur des additions n'est que minime. Mais cette méthode demande beaucoup de pratique. Le résultat du dosage était le suivant : I ml d'une dilution ( I : Io ooo) de bleu de méthylène correspond à $0,005 \mathrm{mg}$ d'acide ascorbique.

La mesure du $\mathrm{pH}$ était faite à $20^{\circ} \mathrm{C}$ avec un appareil Orion dont l'électrode de référence était une électrode au calomel et l'électrode de mesure une électrode à la quinhydrone.

8) Les recherches organoleptiques sur les boissons ont été faites avec l'aide d'une commission de cinq membres (12). Ces derniers ont donné séparément leur appré-

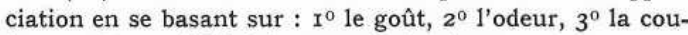
leur, $4^{\circ}$ la possibilité de "popularisation ».

\section{Examen des feuilles de matières plastiques.}

I) Perméabilité à la lumière. Nous avons mesuré avec un densitomètre optique la lumière passant à travers la feuille de plastique. De cette façon nous avons pu savoir dans quelle mesure les feuilles empêchent le passage de la lumière qui favorise la décomposition des vitamines.

2) Perméabilité à la vapeur d'eau : faite suivant les normes (13). 


\section{Lu dans Fruits, il y a 50 ans ...}

3) Perméabilité à l'air. Mesure faite avec un appareil hollandais (Beck's Prinzip) par $65 \%$ d'humidité et $22^{\circ} \mathrm{C}$ (14).

4) L'analyse de la perméabilité au gaz carbonique a été faite dans le récipient utilisé pour mesurer la perméabilité à la vapeur d'eau. Pour constater la quantité et la concentration de la solution nécessaire à la mesure nous avons procédé à des essais préalables (15). Dans les récipients à examiner nous avons mis une solution de $\mathrm{Ba}(\mathrm{OH})_{2}$ à des concentrations et en quantités diverses et nous avons placé ceux-ci dans un dessiccateur à vide sous un jet de gaz carbonique. Pour établir le temps de mensuration nous avons titré un à un les récipients toutes les demi-heures.

5) Perméabilité à la graisse. Elle a été mesurée suivant les prescriptions d'Oswin à $20^{\circ} \mathrm{C}(\mathrm{I} 6)$.

6) Examen des composés extractibles en milieu acide. Nous avons préparé des solutions d'acide citrique à des $\mathrm{pH}$ de 2 à 4 qui concordent avec la teneur en acide des boissons de défense destinées à être entreposées. De chaque feuilie une parcelle d'un gramme fut découpée en petits morceaux, mise dans $100 \mathrm{ml}$ de la solution et agitée pendant 24 heures

FIGUAE 1 - PERMEABILITÉ AU CO2 DE QUELQUES FEUILLES DE MATIËRES PLASTIQUES $\dot{A} 20^{\circ} \mathrm{C}$.

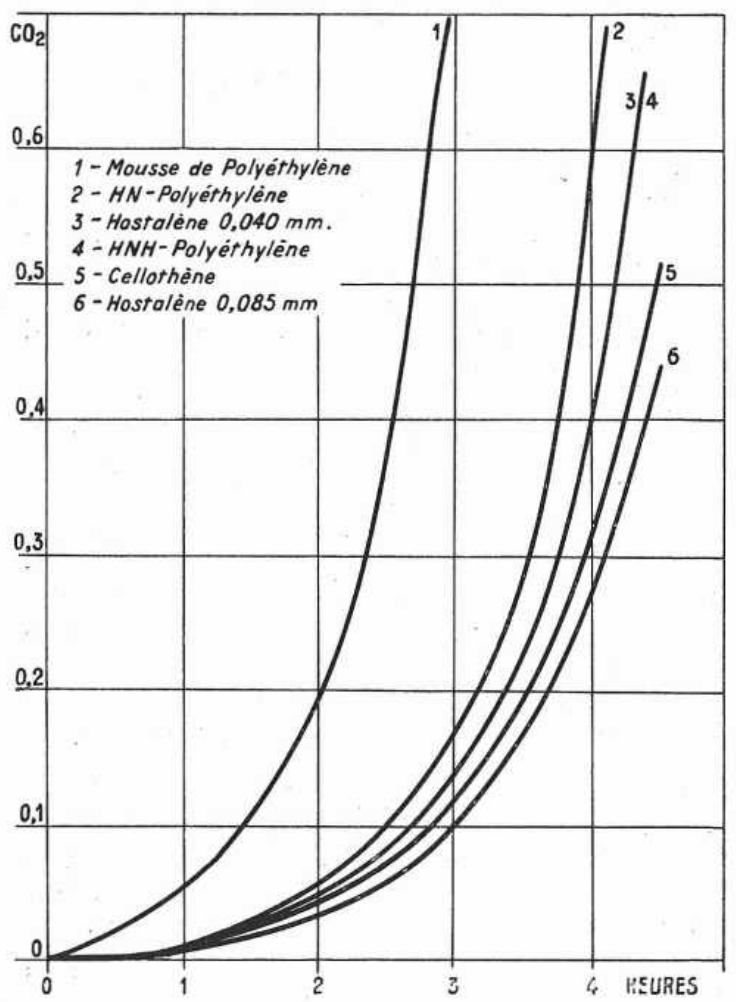

à $20^{\circ} \mathrm{C}$ dans un agitateur à secousses. Ensuite la solution fut examinée au photomètre par rapport à la solution d'acide citrique pure. On nota également les changements de poids des feuilles lavées et séchées.

7) Possibilités de stérilisation. La tolérance à la chaleur des feuilles de plastique fut examinée dans un autoclave à 100-120-135 et $145^{\circ}$ (I-2-3-4 atmosphères).

Une condition indispensable pour l'emballage pratique de certaines boissons de défense particulièrement agréables à consommer chaudes (comme par ex. la soupe au babeurre chaude) est la possibilité de les chauffer légèrement. D'où la nécessité d'examiner également la tolérance à la chaleur.

8) La tolérance au froid de la feuille plastique fut mesurée à $+5^{\circ}$ et $-15^{\circ} \mathrm{C}$.

9) Des recherches sur la possibilité de souder les matières plastiques furent faites au moyen d'un appareil à souder de $220 \mathrm{~V}$ à courant alternatif.

ro) Étude de la solidité. Ces contrôles furent faits au moyen de chutes de $1,50 \mathrm{~m}$ renouvelées jusqu'à ce que les colis contenant les liquides crèvent le long de la ligne de soudure (ou ailleurs).

II) Fixation des chiffres au colorimètre d'après Livibond. Nous avons mesuré au colorimètre les changements de couleur des boissons rouges, jaunes, vertes, et pendant 'entreposage.

\section{Essais d'emballage et d'entreposage.}

Les essais d'emballage et d'entreposage n'ont été réalisés que sur les boissons dont le succès en raison des examens organoleptiques semblait probable. C'est en tenant compte des qualités connues des matières plastiques que fut choisie la feuille convenant à chaque boisson. Puis les boissons furent entreposées dans des récipients en forme de tétraèdre par $+5^{\circ}$ et $-15^{\circ} \mathrm{C}$.

Interprétation des résultats du contrôle.

Dosage du degré d'acidité. Nous avons constaté que l'appareil de titration à haute fréquence n'est utilisable dans l'industrie du lait qu'avec des modifications, car nous ne pouvions assurer l'exactitude des mesures qu'après avoir éliminé des produits laitiers à analyser les graisses et les protides. La lenteur d'un tel procédé constitue un inconvénient de l'appareil.

Dosage de la vitamine $C$. Les résultats permettent de constater qu'après un certain nombre d'essais indispensables la méthode Effer au bleu de méthylène peut être utilisée avec certitude. Cependant, pour avoir des résultats précis, il convient de mener parallèlement aux examens un contrôle avec une solution d'un titre connu d'acide ascorbique. 


\section{Lu dans Fruits, il y a 50 ans}

Recherche du pH. A l'occasion des recherches sur le pH une corrélation fut établie entre l'effet rafraîchissant et la valeur du $\mathrm{pH}$ dans chaque cas. Par ailleurs il a été constaté qu'après un entreposage de 5 à Io jours, la valeur du $\mathrm{pH}$ avait légèrement diminué, aussi bien chez les boissons stockées à $+5^{\circ}$ que che $z$ celles entreposées à $-I 5^{\circ} \mathrm{C}$.

Examens organoleptiques. De ces résultats il apparaît que parmi les 28 boissons composées énumérées dans cet article les ro premières peuvent facilement être diffusées comme boissons de défense d'un goût agréable, d'une grande valeur nutritive et d'une forte teneur en vitamines. Le classement des boissons de défense est indiqué dans le tableau $\mathrm{I}$.

Contrôle des emballages.

Interprétation des essais sur les feuilles plastiques.

Perméabilité à la vapeur d'eau. En se basant sur les données obtenues on peut constater que la feuille en hostalène de $0,085 \mathrm{~mm}$ d'épaisseur présente à $50 \%$ d'humidité et à $20^{\circ} \mathrm{C}$ la perméabilité la plus parfaite.

Perméabilité aux matières grasses. Les feuilles plastiques utilisées pour les essais se sont révélées après 7 jours d'essais comme imperméables à 1'huile à l'exception de la feuille cellulosique de fabrication locale que nous avons utilisée comme élément de comparaison et qui au troisième jour par $20^{\circ} \mathrm{C}$ est apparue comme perméable à 1'huile.

Perméabilité à la lumière. Entre la perméabilité à la lumière et 1'entreposage aucune relation ne peut être établie, les feuilles à perméabilité plus ou moins grande s'étant révélées également bonnes pendant 1'entreposage.

\section{TABLEAU 2.}

Perméabilité à l'air de quelques feuilles de plastique à $22^{\circ} \mathrm{C}$.

\begin{tabular}{|c|c|c|}
\hline Désignation & $\begin{array}{l}\text { Épaisseur } \\
\mathrm{mm}\end{array}$ & $\begin{array}{l}\text { Perméabilizé } \\
\mathrm{ml} / \mathrm{sec} . \mathrm{cm}^{2}\end{array}$ \\
\hline 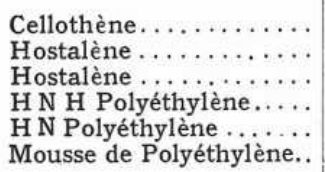 & $\begin{array}{l}0,070 \\
0,085 \\
0,040 \\
0,070 \\
0,030 \\
0,350\end{array}$ & $\begin{array}{l}5,9-10^{4} \\
1,0-10^{6} \\
3,0-10^{5} \\
1,0-10^{3} \\
2,0-10^{3} \\
1,0-10^{1}\end{array}$ \\
\hline
\end{tabular}

Cela est également vrai pour la relation entre l'entreposage et l'épaisseur des feuilles.

Perméabilité à l'air et au gaz carbonique. Les feuilles se sont comportées vis-à-vis de l'air et du gaz carbonique d'une façon égale quelles qu'aient été leurs qualités sélectives (tableau 2 et figure I).

Solubilité aux acides. A l'exception du cellothène les feuilles n'ont pas laissé apparaître de composants solubles après avoir trempé pendant 24 heures dans une solution d'acide citrique à $\mathrm{pH} 2$ et 4 .

Tolérance à la chaleur. Les essais ont montré qu'à $\mathrm{I}^{\circ} \mathrm{C}$ toutes les feuilles sont stérilisables. Le H. N. polyéthylène ne l'est plus à $120^{\circ}, \mathrm{le} \mathrm{H}$. N. H. polyéthylène à $135^{\circ}$ et toutes les autres feuilles ne le sont plus à $145^{\circ}$.

Stabilité au froid. A ce point de vue ce sont les feuilles de mousse de polyéthylène et de cellothène qui convenaient le mieux. Les changements de couleur pendant l'entreposage ont été indiqués au tableau 3 et chiffrés après lecture au colorimètre. A la suite des essais on constate que les boissons colorées artificiellement présentent la plus forte diminution de couleur.

Étude de la résistance. Les emballages en mousse de polyéthylène en cellothène et en $\mathrm{H}$.N. H. polyéthylène sont les plus résistants. Les emballages en hostalène et en H. N. polyéthylène en $0,085 \mathrm{~mm}$ et celles en hostalène en $0,040 \mathrm{~mm}$ sont nettement moins solides (tableau IV).

TABLEAU 4 .

Solidarité des feuilles de plastique (*).

\begin{tabular}{l|c|c}
\hline & \multicolumn{2}{|c}{ Défectuosité } \\
\cline { 2 - 3 } Désignation & partielle & totale \\
& & \\
\hline Hostalène $0,040 \mathrm{~mm} . .$. & 3 & 4 \\
H N Polyéthylène ...... & 4 & I0 \\
Hostalène o,085 mm.... & 5 & II \\
H N H Polyéthylène..... & II & 22 \\
Cellothène............. & I6 & 40 \\
Mousse polyéthylène.... & 25 &
\end{tabular}

(*) Ces chiffres représentent le nombre de paquets s'ouvrant après une chute de $1,50 \mathrm{~m}$. 


\section{Lu dans Fruits, il y a 50 ans}

D'après les résultats des essais et leur interprétation on peut faire les constatations suivantes :

I. En utilisant le lait et les sous-produits du lait on peut fabriquer des boissons de défense répondant à toutes les exigences modernes, bien emballées et facilement entreposables (tableau V).

2. Le goût peu apprécié du petit lait et du babeurre peut être couvert par des mélanges appropriés au goût du jour sans que les qualités favorables des sous-produits laitiers (teneur en sel, acides, etc.) disparaissent.

3. Cette constatation s'applique aussi au lait dont le goût et la composition considérée comme biologiquement parfaite puissent être augmentés de telle façon que la valeur nutritive devienne nettement plus élevée.

4. A la suite des essais sur la saveur et des résultats des études on peut constater que cette composition des boissons de défense ( $\mathrm{I} 7$ ) convient aussi bien aux ouvriers adultes qu'aux jeunes ouvriers et aux élèves des écoles professionnelles (tableau VI).

On conseille :

a) Lait aux amandes, aux châtaignes, aux œufs : dans les écoles en plein air, les maisons de repos pour enfants, les maisons de santé pour enfants malades ou convalescents.

b) Lait aux fruits, aux flocons, petit lait et babeurre aux légumes: surtout pour les jeunes.

c) Soupes chaudes au babeurre : pour les travailleurs sur un lieu de travail non chauffé.

d) Petit lait au citron, boissons au babeurre avec fines herbes : pour les travailleurs dans des lieux de travail chauffés et dans l'agriculture.

TABLEAU No 1

RESULTATS DES EXAMENS ORGANOLEPTIQUES

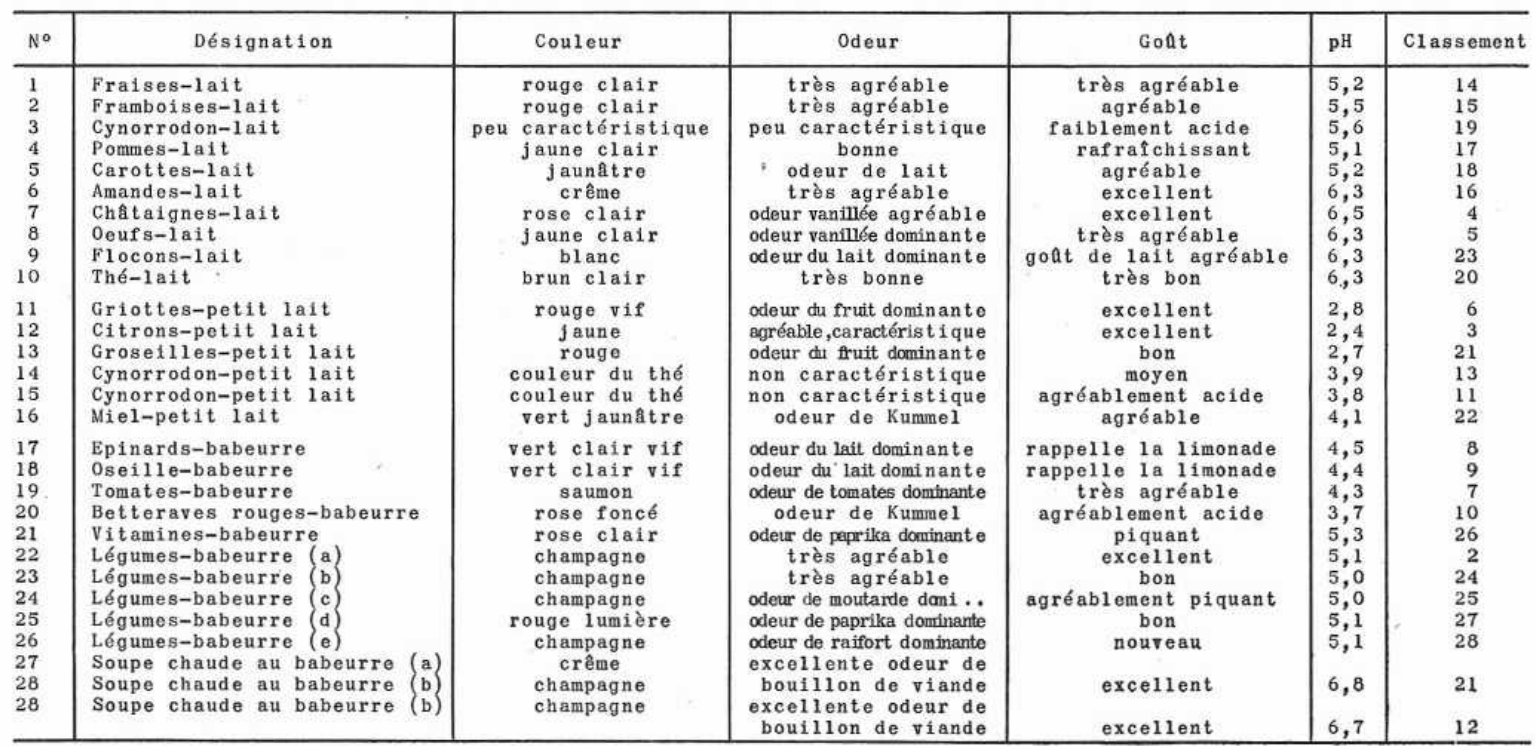


Lu dans Fruits, il y a 50 ans

TABLEAU $N^{\circ} 3$

CHIFFRES AU COLORIMETRE POUR DIVERSES BOISSONS DE DEFENSE

\begin{tabular}{|c|c|c|c|c|c|c|c|c|c|c|c|}
\hline \multirow[b]{2}{*}{ Désignation des boissons } & \multicolumn{5}{|c|}{ Observation } & \multicolumn{5}{|c|}{ Interprétation numérique } & \multirow{2}{*}{$\begin{array}{c}\text { Couleur correspondant } \\
\text { à l'interprétation } \\
\text { numérique } \\
\end{array}$} \\
\hline & Rouge & Jaune & Bleu & B1 anc & Mat & Rouge & Orange & Jaune & Vert & Bleu & \\
\hline $\begin{array}{l}\text { Citrons-petit lait } \\
\text { Griottes-petit lait } \\
\text { Epinards-babeurre } \\
\text { Oseille-babeurre } \\
\text { Betterave rouge-babeurre } \\
\text { Légumes-babeurre } \\
\text { Tomates-babeurre }\end{array}$ & $\begin{array}{r}17,0 \\
1,5 \\
21,0 \\
2,0 \\
8,1\end{array}$ & $\begin{array}{r}10,0 \\
13,1 \\
20,0 \\
4,0 \\
10,1\end{array}$ & $\begin{array}{l}0,4 \\
3,0 \\
4,3 \\
3,2\end{array}$ & $\overline{A V A}$ & $\begin{array}{l}1,5 \\
3,2\end{array}$ & $\begin{array}{l}17,0 \\
21,0\end{array}$ & $\begin{array}{l}2,0 \\
4,9\end{array}$ & $\begin{array}{r}9,6 \\
10,1 \\
12,9 \\
2,0 \\
2,0\end{array}$ & $\begin{array}{l}0,4 \\
3,0 \\
2,8\end{array}$ & & $\begin{array}{c}\text { vert jaunâtre } \\
\text { rouge } \\
\text { vert jaunâtre } \\
\text { vert jaunatre } \\
\text { rouge } \\
\text { orange jaunatre } \\
\text { orange jaunâtre }\end{array}$ \\
\hline \multicolumn{12}{|c|}{ APRES L'ENTREPOSAGE } \\
\hline $\begin{array}{l}\text { Citrons-petit lait } \\
\text { Griottes-petit lait } \\
\text { Epinards-babeurre } \\
\text { Oseille-babeurre } \\
\text { Betterave rouge-babeurre } \\
\text { Légumes-babeurre } \\
\text { Tomates-babeurre }\end{array}$ & $\begin{array}{r}19,0 \\
2,0 \\
4,8\end{array}$ & $\begin{array}{l}8,0 \\
1,1 \\
0,6 \\
2,3 \\
0,3\end{array}$ & 0,5 & - & & $\begin{array}{r}15,0 \\
19,0 \\
4,5\end{array}$ & $\begin{array}{l}2,0 \\
0,3\end{array}$ & $\begin{array}{l}7,5 \\
1,1 \\
0,6 \\
0,3\end{array}$ & 0,5 & & $\begin{array}{c}\text { vert jaunâtre } \\
\text { rouge } \\
\text { jaunatre } \\
\text { jaunâtre } \\
\text { rouge } \\
\text { orange jaunatre } \\
\text { orange jaunâtre }\end{array}$ \\
\hline
\end{tabular}

TABLEAU N० 5

RESULTATS DES ESSAIS D'ENTREPOSAGE

\begin{tabular}{|c|c|c|c|c|c|c|c|}
\hline $\begin{array}{l}\text { Désignation de } \\
\text { la boisson }\end{array}$ & Matière plastique & $\begin{array}{c}\text { Durée d'entreposage } \\
\text { en jours }\end{array}$ & $\begin{array}{c}\text { Température } \\
\text { d'entreposage } \\
{ }^{\circ} \mathrm{C}\end{array}$ & $\begin{array}{l}\text { Perte de } \\
\text { poids } \\
\%\end{array}$ & \multicolumn{2}{|c|}{$\begin{array}{l}\text { Valeur du pH } \\
\text { avant et après } \\
\text { l'entreposage }\end{array}$} & $\begin{array}{c}\text { Appréciation } \\
\text { organoleptique }\end{array}$ \\
\hline $\begin{array}{l}\text { Oeufs-1ait } \\
\text { Châtaignes-1ait }\end{array}$ & $\begin{array}{c}\text { Hostalène } \\
\text { Mousse de polyéthylène }\end{array}$ & $\begin{array}{l}10 \\
10\end{array}$ & $\begin{array}{l}-15^{\circ} \\
-15^{\circ}\end{array}$ & $\begin{array}{l}0,02 \\
0,02\end{array}$ & $\begin{array}{l}6,3 \\
6,5\end{array}$ & $\begin{array}{l}6,1 \\
6,4\end{array}$ & $\begin{array}{l}\text { bonne } \\
\text { bonne }\end{array}$ \\
\hline $\begin{array}{l}\text { Griottes-petit } \\
\text { lait } \\
\text { Citron-petit } \\
\text { lait }\end{array}$ & $\begin{array}{l}\text { Cellothène } \\
\text { Cellothène }\end{array}$ & $\begin{array}{l}5 \\
5\end{array}$ & $\begin{array}{l}+5^{\circ} \\
+5^{\circ}\end{array}$ & $\begin{array}{l}0,27 \\
0,31\end{array}$ & $\begin{array}{l}2,8 \\
2,4\end{array}$ & $\begin{array}{l}2,6 \\
2,1\end{array}$ & $\begin{array}{l}\text { agréable } \\
\text { agréable }\end{array}$ \\
\hline $\begin{array}{l}\text { Epinards-babeurre } \\
\text { Oseille-babeurre } \\
\text { Betterave rouge- }\end{array}$ & $\begin{array}{l}\text { Cellothène } \\
\text { Cellothène }\end{array}$ & $\begin{array}{l}5 \\
5\end{array}$ & $\begin{array}{l}+5^{\circ} \\
+5^{\circ}\end{array}$ & $\begin{array}{l}0,11 \\
0,13\end{array}$ & $\begin{array}{l}4,5 \\
4,4\end{array}$ & $\begin{array}{l}4,2 \\
4,3\end{array}$ & $\underset{\|}{\text { inchangée }}$ \\
\hline $\begin{array}{l}\text { babeurre } \\
\text { Tomates-babeurre } \\
\text { Soupe chaude au }\end{array}$ & $\begin{array}{l}\text { Cellothène } \\
\text { H.N. Polyéthylène }\end{array}$ & $\begin{array}{l}5 \\
5\end{array}$ & $\begin{array}{l}+5^{\circ} \\
+5^{\circ}\end{array}$ & $\begin{array}{l}0,32 \\
0,12\end{array}$ & $\begin{array}{l}3,7 \\
4,3\end{array}$ & $\begin{array}{l}3,6 \\
3,9\end{array}$ & plus "acide \\
\hline $\begin{array}{cc}\text { babeurre } & \text { a) } \\
\text { Soupe chaude au }\end{array}$ & Hostalène & 10 & $-15^{\circ}$ & 0,01 & 6,8 & 6,6 & très agréable \\
\hline babeurre b) & H.N.H. Polyéthylène & 10 & $-15^{\circ}$ & 0,03 & 5,1 & 5,0 & agréable \\
\hline
\end{tabular}

TABLEAU $N^{\circ} 6$

POSSIBILITES D'EMPLOI DES NOUVELLES BOISSONS DE DEFENSE DANS DES GROUPES DE TRAVAIL ET D'AGE DIVERS

\begin{tabular}{|c|c|c|c|c|c|c|c|c|c|}
\hline \multirow{2}{*}{$\begin{array}{c}\text { Groupes de travail } \\
\text { et d'âge }\end{array}$} & \multirow{2}{*}{ kcal } & \multicolumn{3}{|c|}{ Besoin quotidien en } & \multirow{2}{*}{ Boisson proposée } & \multicolumn{4}{|c|}{$4 \mathrm{~d} l$ contiennent en } \\
\hline & & $\begin{array}{c}\text { Vit.c } \\
\text { mg }\end{array}$ & $\underset{\text { mg }}{\text { Vit. } B_{1}}$ & $\underset{\mathrm{mg}}{\mathrm{Vit} . \mathrm{B}_{2}}$ & & $\underset{\mathrm{mg}}{\mathrm{kcal}}$ & $\underset{\mathrm{mg}}{\mathrm{Vit} . \mathrm{C}}$ & $\underset{\text { mg }}{\text { vit. } B_{1}}$ & $\underset{\text { mg }}{\text { Vit. } B_{2}}$ \\
\hline Enfants de $1-2$ ans & 1200 & 50 & 0,75 & 1,00 & F1ocons-1aft & 427,8 & 5,78 & 0,20 & 0,72 \\
\hline Enfants de $3-4$ ans & 1400 & 50 & 0,80 & 1,20 & $\begin{array}{l}\text { Pommes-1ait } \\
\text { Carottes-1ait }\end{array}$ & $\begin{array}{l}332,0 \\
295,2\end{array}$ & $\begin{array}{r}10,28 \\
6,90\end{array}$ & $\begin{array}{l}0,19 \\
0,24\end{array}$ & $\begin{array}{l}0,58 \\
0,58\end{array}$ \\
\hline Enfants de $5-6$ ans & 1600 & 60 & 0,85 & 1,20 & Cynorrhodons-1 ait & 369,0 & 421,22 & 0,20 & 0,67 \\
\hline Enfants de 7-10ans & 1900 & 60 & 1,00 & 1,30 & Oenfs-1ait & 420,8 & 5,94 & 0,23 & 0,92 \\
\hline Enfants del1-12ans & 2400 & 65 & 1,20 & 1,50 & Châtaignes-1ait & 733,0 & 4,12 & 0,29 & 0,79 \\
\hline Enfants del3-15ans & 2800 & 70 & 1,30 & 1,60 & Amandes-1 ait & 773,0 & 4,96 & 0,92 & 2,14 \\
\hline Enfants de16-20ans & 3100 & 75 & 1,50 & 1,80 & Miel-petit lait & 279,2 & 3,40 & 0,16 & 0,37 \\
\hline Apprentis 18-24 ans & 3300 & 90 & 1,70 & 2,00 & Tomates-babeurre & 145,6 & 51,10 & 0,22 & 0,31 \\
\hline Etudiants & 3350 & 80 & 1,60 & 1,90 & $\begin{array}{l}\text { Thé-lait } \\
\text { norrhodons-petit lai }\end{array}$ & $\begin{array}{l}341,0 \\
230,6\end{array}$ & $\begin{array}{r}5,34 \\
418,16\end{array}$ & $\begin{array}{l}0,16 \\
0,19\end{array}$ & 0,65 \\
\hline Travall léger & 2800 & 70 & 1,50 & 1,80 & Citrons-petit lait & 312,2 & 34,90 & 0,16 & 0,28 \\
\hline Travail moyen & 3200 & 80 & 1,80 & 2,10 & Oseille-babeurre & 129,4 & 88,00 & 0,17 & 0,78 \\
\hline & 5000 & . & 1,0 & $2,+0$ & Epinards-babeurre & 111,4 & 90,00 & 0,21 & 0,68 \\
\hline Travail de force & 4500 & 90 & 2,00 & 2,20 & $\begin{array}{l}\text { Soupe chaude au babeurre a } \\
\text { Soupe chaude au babeurre b }\end{array}$ & $\begin{array}{l}315,4 \\
230,7\end{array}$ & $\begin{array}{r}6,00 \\
14,40\end{array}$ & $\begin{array}{l}0,36 \\
1,63\end{array}$ & $\begin{array}{l}0,80 \\
0,63\end{array}$ \\
\hline
\end{tabular}




\section{Lu dans Fruits, il y a 50 ans}

\section{BIBLIOGRAPHIE}

I. BRUnKe R. Milchwirtschaft und Dauermilchindustrie, Leipzig, 1958.

2, Zajkovszkij. Ja. Sz. Physik und Chemie der Milch und Milchprodukte, Budapest, I953.

3. LANG K. Physiologische Beurteilungsliste von Kunststoff-Folien,Wiesbaden-Berlin, 1957.

4. Shulz M. E. - Drache K. D. Milchwiss. 2, 276, I947, ref. ZUL. $88,556,1948$.

5. HESSE A. ZUL. 88,499 , 1948 .

6. Ibidem. 501 .

7. PUNGor E. Hochfrequenz-Titrimeter, Budapest, I960.

8. LÁszirity R.-Nedelkovits J. Élelmiszervizsg. Közl, 6, п1о I960.
9. Berndorfer A.-né. Diplomarbeit, Budapest, 1959.

ro. Kiszel J.-né-Lányi Gy.-né. Elejmezési Ipar 8, III, I954.

II. Wibberg B. ZUL. 76, $128,1938$.

I2. Szilas E.-né-Berndorfer A.-né, Élelmiszervizsg. Közl. 5, I5I, 1959.

I3. Astm. Designation. E 96-53 T. r953.

14. Schrofer W. Kunststoffe 4, I43, 1956.

I5. ERdey L.-Rády Gy. Gázelemzés, Budapest, 1957.

I6. Oswin C. R. Protective Wrappings, London, 1954.

17. TARJÁn R.-Lindner K. Tápanyagtáblázat. 4, Kiadás Budapest, IC59.

Traduit de l'allemand par J. Bur. 